\title{
RESOLVING PRIVACY CONFLICT FOR MAINTAINING PRIVACY POLICIES IN ONLINE SOCIAL NETWORKS
}

\author{
Fulpagare Priya K. and Dr. Nitin N. Patil \\ Department of Computer Engineering, R. C. Patel Institute of Technology, Shirpur
}

\begin{abstract}
In recent years, the use of online social networks (OSNs) such as Facebook, Twitter etc has tremendous increased. Users see these OSNs as a useful tool to find friends and interact with them. Moreover, OSNs allow their users to share photos, videos, and express their thoughts, views and feelings. However, users are usually concerned about their privacy when using OSNs. These OSNs not only offer smart resources for virtual social interfaces and sharing of data but also improve a number of security and privacy issues. While OSNs allow a single user to manage access to her/his data, those currently do not provide any mechanism to apply privacy concerns over data associated with multiple users, remaining privacy harms largely unresolved and leading to the potential confession of information that at least one user planned to keep private. This is because the unrestricted image of a subject can be affected by photos or comments posted on a social network. In this way, recent studies shows that users are demanding better mechanisms to protect their privacy. For this concern, we provide a systematic mechanism to identify and resolve privacy conflicts in online social networks (OSNs). The first computational tool to resolve conflicts for multiparty privacy management in social media. This makes it enable to adapt different situations by modelling the concessions that users make to reach a solution to the conflicts. Our conflict resolution specifies a tradeoff between privacy protection and data sharing by computing privacy risk and sharing loss.
\end{abstract}

Key words: Online social networks, Social media; Content sharing sites, Privacy, Privacy preserving, Conflicts resolution.

Cite this Article: Fulpagare Priya K. and Dr. Nitin N. Patil, Resolving Privacy Conflict for Maintaining Privacy Policies in Online Social Networks, International Journal of Computer Engineering and Technology 10(3), 2019, pp. 94-101. http://iaeme.com/Home/issue/IJCET?Volume=10\&Issue=3

\section{INTRODUCTION}

Online social networks (OSNs), such as Facebook, Twitter, and Google+, have become an actual portal for hundreds of millions of Internet users. For example, Facebook, one of representative social network provider, claims that it has more than 800 million active users. With the help of these OSNs, people share personal and public information and make social 
connections with friends, coworkers, colleagues, family and even with strangers. As a result, OSNs store a huge amount of possibly sensitive and private information on users and their interactions. To defend that information, privacy control has been carried out as a central feature of OSNs. OSNs provide in-built mechanisms allowing users to interconnect and share information with other members [1].

A typical OSN offers each user with a practical space containing:

- Profile data,

- A list of the user's friends, and

- Web pages such as wall in Facebook, where the user and friends can post content and leave messages.

A user profile typically includes information with respect to the user's birthday, gender, interests, education and work history, and contact information. In addition, users can not only upload a content into their own or other's spaces but also tag other users who appear in the content. Each tag is an unambiguous situation that links to a user's space. For the security of user data, current OSNs ultimately require users to be system and policy administrators for variable their data, where users can restrict data sharing to a specific set of trusted users. OSNs frequently use user relationship and group membership to distinguish between authorized and unauthorized users. For example, in Facebook, users can allow friends, friends of friends, specific groups or everyone to access their data, relying on their personal privacy requirements. Although the fact that OSNs currently provide privacy control mechanisms allowing users to regulate access to information contained in their own spaces, users, unfortunately, have no control over data residing outside their spaces. For example, if a user posts a comment in a friend's space, he/she cannot specify which users can view the comment. In another case, when a user uploads a photo and tags friends who seem in the photo, the tagged friends cannot control who can see this photo. Since multiple associated users may have unlike privacy concerns over the shared data, privacy conflicts occur and the lack of shared privacy control increases the possible risk in leaking sensitive information by friends to the public [2].

Users in OSNs can post statuses and notes, upload photos and videos in their own spaces, tag others to their content, and share the content with their friends. On the other hand, users can also post information in their friend's spaces. The mutual content may be connected with multiple users. Example: Consider an example where a photograph contains three users, A, B and $\mathrm{C}$. If $\mathrm{A}$ uploads it to their own space and tags both $\mathrm{B}$ and $\mathrm{C}$ in the photo, in this sense we say $\mathrm{A}$ is the owner of the photo, and B and $\mathrm{C}$ stakeholders of the photo. All of them may be preferred to specify privacy policies to control over who can see this photo. In another case, when A posts a note stating "I will attend a party on Friday night with $@$ C" to B's space, we say $\mathrm{A}$ is the contributor of the note and he/she may want to make the control over her/his notes. In addition, since $C$ is explicitly identified by at mention in this note, he/she is considered as a stakeholder of the note and may also want to control the publicity of this note. Since each associated user may have dissimilar privacy concerns over the mutual content, privacy conflicts can occur among the multiple users. OSNs also enable users to share others' content. For example, when A views a photo in B's space and decides to share this photo with their friends, the photo will be in turn posted to A's space and A can authorize his/her friends to see this photo. In this case, $\mathrm{A}$ is a disseminator of the photo. Since A may adopt a weaker control saying the photo is visible to everyone, the initial privacy concerns of this photo may be violated, resulting in the leakage of sensitive information during the procedure of data dissemination. Figure 1 shows a broad conflict scenario in content sharing where the sharing starts with a contributor who uploads the content, and then a disseminator views and shares 
the content. All privacy conflicts among the disseminator and the original controllers (the owner, the contributor and the stakeholders) should be taken into account for regulating access to content in disseminator's space. In addition to privacy conflicts in content sharing, conflicts may also occur in two other situations, profile sharing and friendship sharing, where multiple parties may have different privacy requirements in sharing their profiles and friendship lists with others or social applications in OSNs.

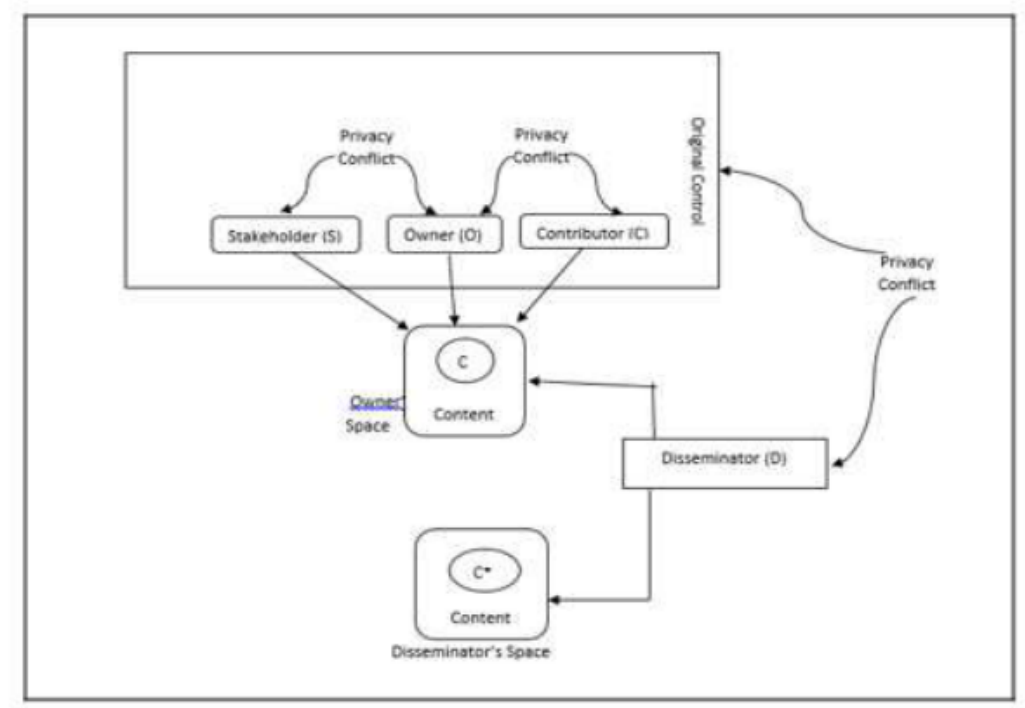

Figure 1 Privacy Conflicts in OSNs.

\section{LITERATURE SURVEY}

In this section, we conduct literature survey of work-done in this topic till now. A literature survey is an independent summary of published research, literature relevant to a topic under thought on a particular topic and may justify future research into a previously overloaded or understudied data. Following are some literature reviews.

Wishart et al. proposed a method to define privacy policies combinedly. In their approach all of the parties involved can define strong and weak privacy preferences. Though, this approach does not involve any computerized technique to solve conflicts, only some recommendations that the users might want to consider when they try to solve the conflicts manually [1].

Shehab et al. described the work which is based on an inducement mechanism where users are satisfied with a quantity of numeracies each time they share information or accept the presence of other users that are co-owners who are affected by the same item. When there are conflicts among co-owners' policies, users can spend their numeracies request for the policy that is best for them. Then, the use of the Clark Tax mechanism is suggested to obtain the highest try [2].

Jorgensen et al. stated that the users may have difficulties to comprehend the mechanism and specify appropriate bid values in auctions. Moreover, users that earned much numeracies in the past will have more numeracies to spend it at will, potentially leading to autonomous decisions. Users must manually define for each item: the privacy settings for the item, their trust to the other users, the understanding of the item, and how much privacy risk they would like to take. These parameters are used to calculate what the authors call privacy risk and sharing loss on segments they define segments as the set of conflicting target users among a set of negotiating users [3]. 
Ahn et al. discussed the voting rules. The authors consider that a different voting rule could be applied depending on the situation. However, it is the user who uploads/posts the item the one who chooses manually which one of the voting rules (UO, MV, VV) to apply for each item. The main problem with this apart from having to specify the voting rule manually for every item is that the choice of the voting rule to be applied is unilateral. That is, the user that uploads the item decides the rule to apply without considering the rest of the negotiating users' preferences, which becomes a unilateral decision on a multi-party setting. Moreover, it might actually be quite difficult for the user that uploads the item to anticipate which voting rule would produce the best result without knowing the preferences of the other users [4].

Rovatsos et al. analyzed the problem of negotiating a solution to multiparty conflicts, from a game-theoretic point of view. These proposals provide an elegant analytic framework proposing negotiation protocols to study the problem and the solutions that can be obtained using well-known game theoretic solution concepts such as the Nash equilibrium [5].

Yang et al. shown the proposals. These proposals may not always work well in practice, as they do not capture the social idiosyncrasies considered by users in the real life when they face multi-party privacy conflicts, and users' behavior is far from perfectly rational as assumed in these game-theoretic approaches [6].

Carminati et al. introduced a hope-based access control mechanism, which allows the specification of access rules for online resources where sanctioned users are denoted in terms of the relationship type, depth, and trust level between users in OSNs. They further presented a semi-decentralized unrestricted access control system and a related application mechanism for controlled sharing of information in OSNs [7].

Liu et al. proposed a framework to compute the privacy score of a user, indicating the user's potential risk caused by her/his participation in OSNs. Their solution also focused on the privacy settings of users with respect to their profile items. Compared with those existing work, our approach measures the privacy risk caused by different privacy concerns from multiple users, covering profile sharing, friendship sharing, as well as content sharing in OSNs [8].

\section{METHODOLOGY}

Current online social networks, such as Facebook, only tolerate the data owner to fully control the shared data, but lack a mechanism to specify and apply the privacy fears from other associated users, important to privacy conflicts being largely unresolved and sensitive information being potentially disclosed to the public. The solution for this conflict incident is the use of mediator.

\section{Conflict Resolution}

When two users disagree on whom the shared data item should be visible to, we say a privacy conflict occurs.

The use of a mediator which detects conflicts, suggests a possible solution to them. Probably in most social media infrastructures, such as Facebook, Twitter, Google + and the like, this mediator could be integrated as the back-end of SM privacy controls' interface; or it could be implemented as a social media application such as a Facebook application that works as an edge to the privacy controls of the original social media organization. :

- The mediator inspects the individual privacy policies of all users for the item and flags all the conflicts found. Basically, it looks at whether individual privacy policies suggest conflicting access control decisions for the same target user. If conflicts are found the item is not shared defensively. 
- The mediator proposes a solution for each conflict found. The mediator estimates how willing each assigning user may be to grant by considering: his/her individual privacy preferences, how sensitive the particular item is for his/her, and the relative importance of the conflicting target users for his/her [3].

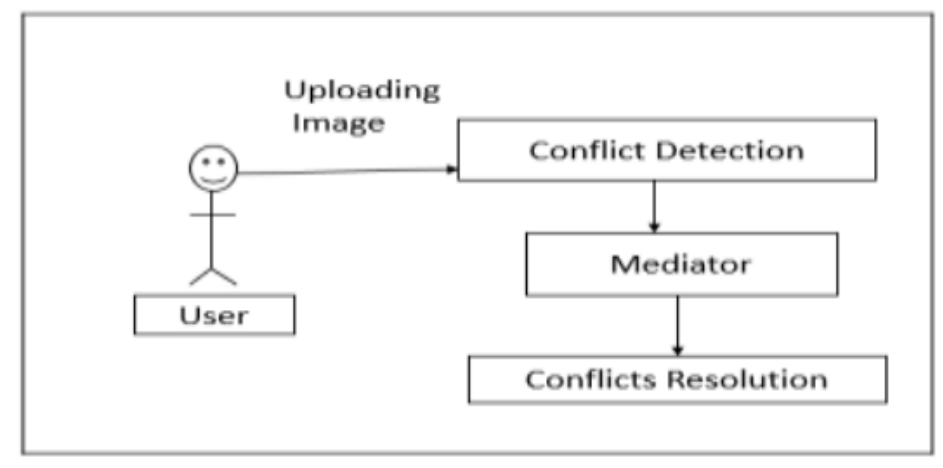

Figure 2 Mechanism Overview.

\section{Computing Conflict Resolution}

Concession Rules: Negotiations about privacy in social media are shared most of the time. That is, users would consider other's inclinations when deciding to whom they share, so users may be willing to grant and change their initial most preferred option. Being able to model the situations in which these concessions occur is of crucial importance to propose the best solution to the conflicts found one that would be acceptable by all the users involved.

Following are user rule for privacy preference of each user, these user rules are used for conflict resolve $[4,5]$ :

I do not mind (IDM) rule: In this rule, if any user want to upload own item in the network at that time another user have no objection on that.

I understand (IU) rule: In this rule, if one user want to share the photo and another one can't share that photo at that time the users do not want to cause any harm to their friends and will normally listen to their objections.

No concession (NC) rule: For the other cases in which neither IDM nor IU applies, then the mediator estimates that a negotiating user would not grant and would prefer to stick to his/her preferred action for the conflicting target user.

The mediator computes the solution for each conflict found by applying the concession rules defined above. If it is not conflicting, the mediator assigns to this target user the action shared by all negotiation users. If $t$ is conflicting, the mediator assigns to its proposal to solve the conflict.

In particular, for each conflicting target user $[6,7]$ :

- If for all negotiating users, their willingness to accept changing their preferred action for the conflicting target user is high, then, according to concession rule IDM, the mediator assumes that all users are willing to grant if need be, so that the final action to be applied for target user $\mathrm{t}$ can be both grating and denying. In order to select one of these two actions, the mediator runs a modified majority voting rule. In precise, this function selects the action that is most favored by the majority of users. In case that there is a tie i.e., the number of users who wish granting and the number of users who wish denying is the same, then the uploaded is given an extra vote.

- If there are users whose willingness to accept changing their preferred action for the conflicting target user is low, then the mediator considers two cases: 
0 if there are at least two users with low willingness and different favored actions, then, according to concession rule IU, the action to be taken should be denying the conflicting target user access to the item in question;

- Otherwise, rule IDM applies so that the users that have high willingness will concede and the user/users who has/have low willingness will determine the action that is finally chosen as the solution.

Though, the specific concession rule instantiated in each situation are rest on the individual privacy policy of each user, the sensitivity of the item for the user, and the relative importance of the conflicting target user, which may vary from participant to participant.

Definition of the Individual Privacy Policy: Each participant was asked to define her/his most preferred privacy policy for each photo.

Conflict and Concession Question: Once the participants defined their individual privacy policy for the photo, a conflict was generated. That is, we told the participants that one or more of the other people in the photo had a different most preferred action for one particular person, specifying the relationship type and strength the participant would have to this person. For instance, if the participant only needed to share the photo with close friends, we told her/him that the other people in the photo needed to share the photo with someone that was her/his friend. Where multiple options were available to generate a conflict, we chose one of them randomly. Then, we asked participants whether or not they would concede and change their most preferred action for that person to solve the conflict with the other people depicted in the photo [8].

\section{EXPERIMENTAL RESULTS}

For the performance evaluation of resolving conflicts for privacy preservation in social media, the system is run on configuration having Windows 7 with 4GB RAM. This method is implemented in JAVA. For this system JSP works on a front end and MySQL on the back end. JSP is used to store all code which we generate in implementation phase. The system implemented as a Java file embedded in an open source content management site, deployed using an Apache server. The analysis of work is supported with the following graph as shown in figure 3. From above discussion it is clear that the existing system is although effective system for circulation or for use but lack of some information creates wrong policies. The $\mathrm{A} 3 \mathrm{P}$ - social and A3P-core are the main aspects of the system. When user uploads the image, the image will be first sent to A3P-core. The work of A3P-core classifies the image and check whether there is a need to invoke the A3P-social. In image classification the image is classified on content based data and metadata based data.

Privacy policies are automatically generated by the system. If this metadata based information is not available, it is difficult to generate accurate privacy policy. This is the drawback of the system to be overcome. This inaccurate privacy creation classification will be the consequence of manual creation of metadata log information.

\section{Analysis of Conflict Resolution}

The results gathered through the existing approach were compared to the results that would have been obtained if our proposed mechanism was applied to the scenarios and if automated voting mechanisms were applied. In particular, we compared the results that would have been obtained applying our proposed mechanism to those that would have been obtained applying the general voting mechanisms used in automated approaches:

Uploader overwrites (UO): The conflict is solved selecting the action chosen by the user that uploads the item. This is the policy currently followed by most Social Media Sites (Facebook, etc.) 
Majority voting (MV): The conflict is solved selecting the action most preferred by the majority of the negotiating users.

Veto voting (VV): If there is one negotiating user whose most preferred action is denying access, the conflict is solved by denying access to the item.

Figure 3 shows the results for each of the above voting mechanisms as well as the results for our proposed mechanism for automated conflict resolution. We can observe that our proposed mechanism ACR (Conflict Resolution) clearly outperformed UO, MV, and VV.

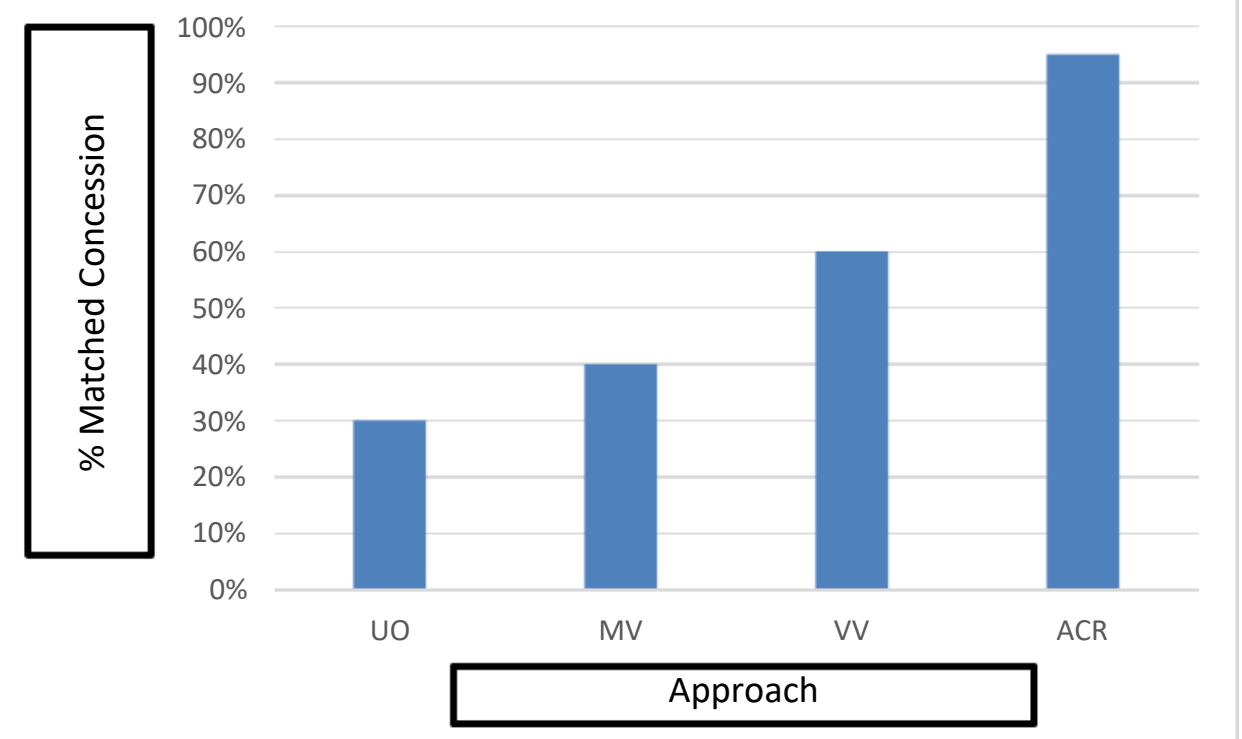

Figure $3 \%$ Graph for Different Voting Mechanisms for Automated Conflict Resolution

Following figure 4 shows us the comparison result of existing approach and proposed approach. In comparison of existing system, the conflict resolution is a technique to avoid the contraction. When inaccurate policies are generated i.e. overlapping of privacy is there, the conflict detection and resolution is required. This conflict resolution technique will improve the efficiency of the existing system.

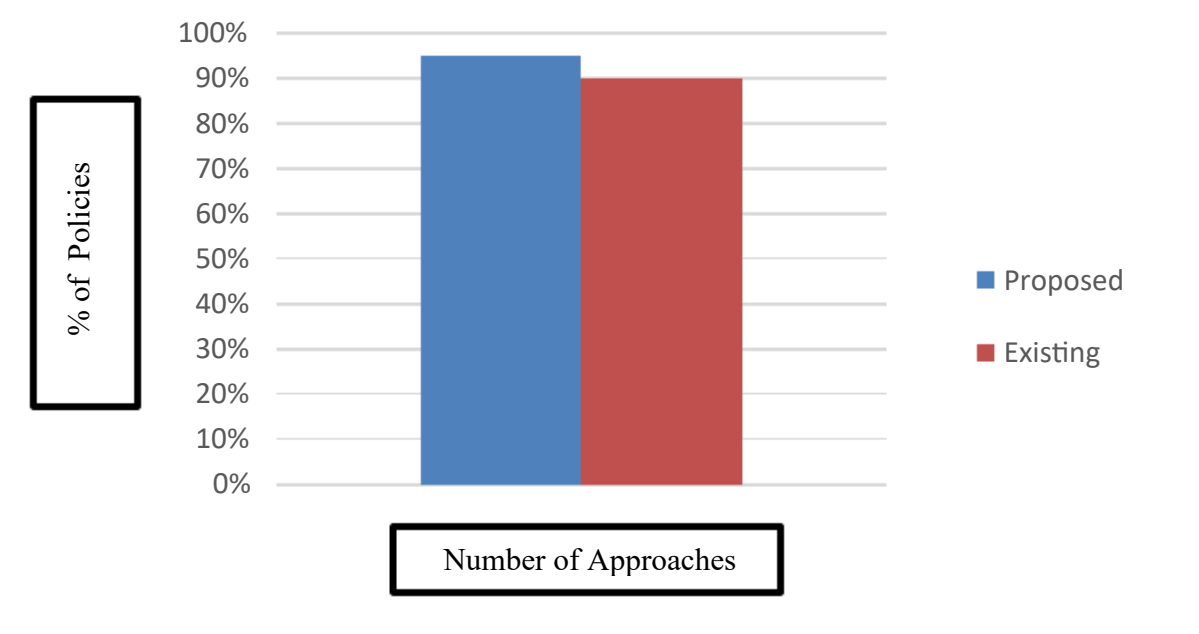

Figure 4 Comparison of Existing and Proposed Approach 


\section{CONCLUSIONS AND FUTURE WORK}

In this paper, we present the first mechanism for detecting and resolving privacy conflicts in Social Media that adapt the conflict resolution strategy based on the particular situation. The mediator firstly examines the individual privacy policies of all users involved looking for possible conflicts. If conflicts are found, the mediator advises a solution for each conflict according to a set of concession rules that model how users would actually convert in this domain. We conducted a user study comparing our mechanism to what users would do themselves in a number of circumstances. The results gained suggest that our mechanism was able to match participant concession behavior significantly, more often than other existing approaches. This has the potential to reduce the amount of manual user interferences to achieve a satisfactory solution or results.

Future research should look into how other aspects could help further increase the precision of the mechanism presented here. The mechanism proposed here are captures general user behavior and would be able to adapt to both different situations and users.

\section{REFERENCES}

[1] R. Wishart, D. Corapi, S. Marinovic, and M. Sloman, "Collaborative privacy policy authoring in a social networking context," in POLICY. IEEE, 2010, pp. 1-8.

[2] A. Squicciarini, M. Shehab, and F. Paci, "Collective privacy management in social networks," in WWW. ACM, 2009, pp. 521-530.

[3] H. Hu, G.-J. Ahn, and J. Jorgensen, "Detecting and resolving privacy conflicts for collaborative data sharing in online social networks," in Proc. ACSAC. ACM, 2011, pp. 103-112. [Online]. Available: http://doi.acm.org/10.1145/2076732.2076747.

[4] H. Hu, G. Ahn, and J. Jorgensen, "Multiparty access control for online social networks: model and mechanisms," IEEE TKDE, 2013.

[5] J. M. Such and M. Rovatsos, "Privacy policy negotiation in social media," ACM Transactions on Autonomous and Adaptive Systems, p. In press. 2015.

[6] H. Hu, G.-J. Ahn, Z. Zhao, and D. Yang, "Game theoretic analysis of multiparty access control in online social networks," in Proceedings of ACM SACMAT '14, 2014, pp. 93102.

[7] B. Carminati, E. Ferrari, and A. Perego, "Rule-based access control for social networks," In on the Move to Meaningful Internet Systems 2006: OTM 2006 Workshops, pages 1734-1744. Springer, 2006.

[8] K. Liu and E. Terzi, "A framework for computing the privacy scores of users in online social networks," ACM Transaction son Knowledge Discovery from Data (TKDD), 5(1):6, 2010. 